\title{
Staying alive : il reste du pain sur la planche !
}

\author{
Staying alive: there is still plenty on our plate!
}

\author{
P. Ray \\ (C) SRLF et Springer-Verlag France 2011
}

Avec une mortalité de près de 50000 personnes par an, une survie à un an sans séquelles neurologiques de moins de $10 \%$, et une incidence de 5 pour 10000 habitants par an, l'arrêt cardiaque (AC) reste un problème de santé publique majeur en France, ainsi que dans les pays occidentaux. Pourtant, de nombreux progrès ont été réalisés ces dernières années et de nombreuses études sur le sujet sont régulièrement publiées dans les plus grands journaux scientifiques [1-7].

Il faut d'ailleurs noter la présence d'importants travaux français parmi ces avancées récentes. Les progrès des dix dernières années concernent autant les médicaments de l'AC et de l'hypothermie, que la diffusion des premiers gestes de secours, la défibrillation semi-automatique par le public et la démonstration de la nécessité de l'adhésion des professionnels de santé (médecins ou paramédicaux) aux recommandations [8].

Ces dernières (nord-américaines ou européennes) insistent maintenant principalement sur l'importance de débuter tôt, et par les témoins, le massage cardiaque externe (MCE), ainsi que sur la nécessité de limiter au maximum son interruption, voire de ne pratiquer initialement que le MCE chez l'adulte pour les AC extrahospitaliers présumés d'origine cardiaque. La réanimation cardiorespiratoire $(\mathrm{RCP})$ par chest compression only (appelée aussi cardiocerebral resuscitation) semble donner des résultats prometteurs $[9,10]$. Les nouvelles recommandations internationales ont d'ailleurs été publiées fin 2010 [2] et insistent sur les points suivants : interrompre le moins possible les compressions thoraciques, ratio entre les compressions et la ventilation de 30 pour 2 , rythme de compression de 100 à 120/min avec des compressions de 5 à $6 \mathrm{~cm}$, pas plus de cinq secondes pour deux insufflations, abandon des deux minutes de RCP si aucun massage n'était en cours avant une défibrillation et apprentissage de l'identification du gasp.

\section{P. Ray $(\square)$}

Service d'accueil des urgences, groupe hospitalier Pitié-Salpêtrière,

UPMC Paris-VI, 47-86, boulevard de l'Hôpital,

F-75013 Paris, France

e-mail : patrick.ray@psl.aphp.fr
Cependant, de nombreuses incertitudes persistent sur la meilleure prise en charge possible des AC extrahospitaliers et des progrès sont encore nécessaires pour en améliorer leur pronostic. En particulier, plusieurs questions restent en suspens :

- en France et en Europe (par rapport au Japon, aux ÉtatsUnis et au Canada), les études épidémiologiques sont encore trop rares (réseau, registre) alors même que sont issues des Samu/Smur français des avancées scientifiques importantes ;

- comment sensibiliser encore davantage les pouvoirs publics français sur l'importance de la diffusion large des défibrillateurs semi-automatiques et de leur utilisation par le grand public ? Une étude récente suggère ainsi que la mise en place de défibrillateurs semi-automatiques au Japon a permis de diminuer la mortalité de l'AC ;

- comment améliorer la formation du public aux gestes de premier secours ? Parmi les pays européens, la France est en retard pour la formation du personnel des entreprises et des administrations, ainsi que du grand public. Malheureusement, les gestes de premier secours sont également mal connus des médecins non rodés à cette pathologie (donc hors urgence, anesthésie, cardiologie et réanimation) et probablement mal ou insuffisamment enseignés et répétés durant les longues années d'étude de médecine. Dans une grande université parisienne, environ $40 \%$ des étudiants en médecine de DCEM2 défibrillaient en première intention un $\mathrm{AC}$ asystolique !

- Quelle est la meilleure prise en charge ventilatoire possible ? Peut-on même s'en passer lors d'un AC extrahospitalier présumé d'origine cardiaque ?

- Les techniques automatisées de MCE ou d'autres matériaux utilisés améliorent-ils le pronostic et quelle méthode choisir $[11,12]$ ?

- L'adrénaline reste-t-elle le meilleur médicament de l'AC ? Bien que méthodologiquement discutable, la publication suédoise récente remet en cause le dogme de l'adrénaline comme premier traitement de l'AC présumé d'origine cardiaque $[6,13]$;

- quand et comment faut-il réaliser l'hypothermie ? Ainsi, la France avec son système préhospitalier médicalisé est 
pionnière en matière d'hypothermie préhospitalière. Pourtant, la démonstration de son utilité sur le pronostic reste à prouver, et surtout les diverses méthodes et techniques utilisées rendent toute comparaison et évaluation scientifique difficile $[13,14]$. Faut-il limiter l'hypothermie aux seuls AC extrahospitaliers d'origine présumée cardiaque ou peut-on l'étendre aux AC intrahospitaliers dont l'origine multifactorielle est beaucoup plus fréquente?

- L'évaluation rapide du pronostic avec son cortège de décision de limitation ou d'arrêt des traitements est fondamentale lorsqu'on connaît la gravité du coma postanoxique et son coût. La création de scores pronostiques nord-américains utilisables en préhospitalier est intéressante dans ce contexte... Mais quid en pratique ? Is it always wrong to perform futile CPR $[15,16]$ ?

- Comment prendre en charge les premiers jours de la phase post-AC avec une défaillance multiviscérale qui s'apparente au sepsis-like? La recherche autour de la réanimation de la phase post-AC se développe depuis relativement peu, les médecins étant toujours à la recherche du Graal pour les médicaments à visée neuroprotectrice [17].

Il est également important de souligner que la prise en charge de l'AC extrahospitalier ne se limite pas à la phase préhospitalière, et seuls un réseau et une transversalité de prise en charge pourront améliorer le pronostic, avec la participation conjointe des urgentistes et des réanimateurs.

\section{Références}

1. Spaulding CM, Joly LM, Rosenberg A, et al (1997) Immediate coronary angiography in survivors of out-of-hospital cardiac arrest. N Engl J Med 336:1629-33

2. Bernard SA, Gray TW, Buist MD, et al (2002) Treatment of comatose survivors of out-of-hospital cardiac arrest with induced hypothermia. N Engl J Med 346:557-63

3. Böttiger BW, Arntz HR, Chamberlain DA, et al (2008) Thrombolysis during resuscitation for out-of-hospital cardiac arrest. N Engl J Med 359:2651-62

4. Kitamura T, Iwami T, Kawamura T, et al (2010) Nationwide public-access defibrillation in Japan. $\mathrm{N}$ Engl J Med 362: 994-1004

5. Gueugniaud PY, David JS, Chanzy E, et al (2008) Vasopressin and epinephrine vs epinephrine alone in cardiopulmonary resuscitation. N Engl J Med 359:21-30
6. Olasveengen TM, Sunde K, Brunborg C, et al (2009) Intravenous drug administration during out-of-hospital cardiac arrest: a randomized trial. JAMA 302:2222-9

7. Nolan JP, Soar J, Zideman DA, et al (2010) European resuscitation council guidelines for resuscitation 2010 section 1. Executive summary. Resuscitation 81:1219-76

8. Hinchey PR, Myers JB, Lewis R, et al (2010) Improved outof-hospital cardiac arrest survival after the sequential implementation of 2005 AHA guidelines for compressions, ventilations, and induced hypothermia: the Wake County experience. Ann Emerg Med 56:348-57

9. Ong ME, Ng FS, Anushia P, et al (2008) Comparison of chest compression only and standard cardiopulmonary resuscitation for out-of-hospital cardiac arrest in Singapore. Resuscitation 78:119-26

10. Bobrow BJ, Spaite DW, Berg RA, et al (2010) Chest compression-only CPR by lay rescuers and survival from outof-hospital cardiac arrest. JAMA 304:1447-54

11. Plaisance P, Lurie KG, Vicaut E, et al (1999) A comparison of standard cardiopulmonary resuscitation and active compressiondecompression resuscitation for out-of-hospital cardiac arrest. French Active Compression-Decompression Cardiopulmonary Resuscitation Study Group. N Engl J Med 341:569-75

12. Duchateau FX, Gueye P, Curac S, et al (2010) Effect of the AutoPulse automated band chest compression device on hemodynamics in out-of-hospital cardiac arrest resuscitation. Intensive Care Med 36:1256-60

13. Ong ME, Tan EH, Ng FS, et al (2007) Survival outcomes with the introduction of intravenous epinephrine in the management of out-of-hospital cardiac arrest. Ann Emerg Med 50:635-42

14. Bruel C, Parienti JJ, Marie W, et al (2008) Mild hypothermia during advanced life support: a preliminary study in outof-hospital cardiac arrest. Crit Care 12:R31

15. Truog RD (2010) Is it always wrong to perform futile CPR? N Engl J Med 362:477-9

16. Morrison LJ, Visentin LM, Kiss A, et al (2006) Validation of a rule for termination of resuscitation in out-of-hospital cardiac arrest. N Engl J Med 355:478-87

17. Neumar RW, Nolan JP, Adrie C, et al (2008) Postcardiac arrest syndrome: epidemiology, pathophysiology, treatment, and prognostication. A consensus statement from the International Liaison Committee on Resuscitation (American Heart Association, Australian and New Zealand Council on Resuscitation, European Resuscitation Council, Heart and Stroke Foundation of Canada, InterAmerican Heart Foundation, Resuscitation Council of Asia, and the Resuscitation Council of Southern Africa); the American Heart Association Emergency Cardiovascular Care Committee; the Council on Cardiovascular Surgery and Anesthesia; the Council on Cardiopulmonary, Perioperative, and Critical Care; the Council on Clinical Cardiology; and the Stroke Council. Circulation 118:2452-83 